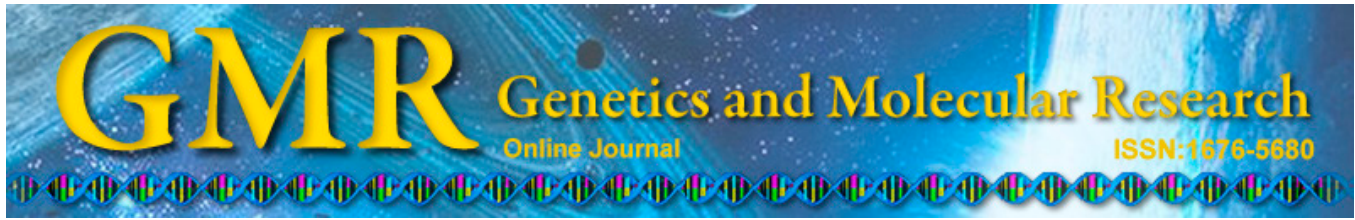

\title{
Improved Agrobacterium-mediated transformation and high efficiency of root formation from hypocotyl meristem of spring Brassica napus 'Precocity' cultivar
}

\author{
X.X. Liu ${ }^{1 *}$, S.R. Lang ${ }^{1 *}$, L.Q. Su, X. Liu and X.F. Wang \\ National Engineering Laboratory for Tree Breeding, \\ College of Biological Sciences and Technology, \\ Beijing Forestry University, Haidian District, Beijing, China \\ *These authors contributed equally to this study. \\ Corresponding author: X.F. Wang \\ E-mail: wxf801@sina.com
}

Genet. Mol. Res. 14 (4): 16840-16855 (2015)

Received January 29, 2015

Accepted May 25, 2015

Published December 14, 2015

DOI http://dx.doi.org/10.4238/2015.December.14.11

\begin{abstract}
Rape seed (Brassica napus L.) is one of the most important oil seed crops in the world. Genetic manipulation of rapeseed requires a suitable tissue culture system and an efficient method for plant regeneration, as well as an efficient transformation procedure. However, development of transgenic B. napus has been problematic, and current studies are limited to cultivated varieties. In this study, we report a protocol for regeneration of transgenic rape after Agrobacterium-mediated transformation of hypocotyls from the spring B. napus 'Precocity' cultivar. We analyzed the effects of plant growth regulators in the medium on regeneration. Additionally, factors affecting the transformation efficiency, including seedling age, Agrobacterium concentration, infection time, and co-cultivation time, were assessed by monitoring GUS expression. Results from these experiments revealed that transformation was optimized when the meristematic parts of the
\end{abstract}


hypocotyls were taken from 8 day-old seedlings, cultured on Murashinge and Skoog basal media containing $0.1 \mathrm{mg} / \mathrm{L}$ 1-naphthaleneacetic acid and $2.5 \mathrm{mg} / \mathrm{L}$ 6-benzylaminopurine, and incubated in Agrobacterium suspension $\left(\mathrm{OD}_{600}=0.5\right)$ for 3 to $5 \mathrm{~min}$, followed by 2 days of cocultivation. Integration of T-DNA into the plant genome was confirmed by polymerase chain reaction (PCR), $\beta$-glucuronidase histochemical staining, and quantitative real-time PCR. The protocols developed for regeneration, transformation, and rooting described in this study could help to accelerate the development of transgenic spring rape varieties with novel features.

Key words: Brassica napus 'Precocity' cultivar; Shoot regeneration; Leaf-disc transformation; GUS histochemical assay

\section{INTRODUCTION}

Due to its economical and nutritional value, Brassica napus is one of the major oilseed crops worldwide (Kong et al., 2011). The customary approaches currently used to improve $B$. napus production, such as cross hybridization, are associated with many problems, including the long juvenile stage and the high degree of heterozygosity, as well as problems with pollen and ovule sterility. However, genetic engineering technologies can be highly valuable for crop improvement, and can lead to the development of novel varieties with improved traits. Therefore, genetic engineering technologies could have a substantial impact on both biotic research and agricultural biotechnology related to B. napus. Plant transformation technologies facilitate integration of a gene into the plant genome, followed by regeneration of the entire plant. Various techniques have been used for transformation in plants, including electroporation, protoplast transfection, and microspore transfection (Hu et al., 1999; Bhalla and Singh, 2008; Abdollahi et al., 2009). However, the Agrobacterium-mediated transformation approach is considered to be superior to other methods owing to its ease of application and low cost. Several studies have reported successful transformation of B. napus with Agrobacterium tumefaciens (Chhikara et al., 2012). These transformation technologies can be used for gradual crop improvement, as they have been used to produce novel improved traits, such as herbicide tolerance (Konagaya et al., 2013), regulated oil composition (Knutzon et al., 1992), insect resistance (Keshamma et al., 2012), and salt tolerance (Dhaka et al., 2013).

In addition to A. tumefaciens-mediated transformation, plant tissue regeneration is a critical process in plant transgenesis. Regeneration depends on many factors, including plant cultivar type, age of explants, media composition, environmental conditions, and hormonal combinations (Bhalla and Smith, 1998; Zhang et al., 2005; Maheshwari et al., 2011). In addition, various types of tissues have been reported to be useful for Agrobacterium-mediated transformation in B. napus, such as cotyledons, stems (Fry et al., 1987; Pua et al., 1987), roots, and hypocotyls (Zhang and Bhalla, 2004; Moghaieb et al., 2006; Mashayekhi et al., 2008).

Halfhill et al. (2001) found that transformation of B. napus (canola) using hypocotyls as explants resulted in only $4 \%$ efficiency. In order to reduce the number of cultivars required to obtain transgenic plants and to provide better transformation efficiencies for other varieties, methods for improvement of transformation efficiency are urgently needed. 
In this study, we describe the development of a complete procedure for Agrobacteriummediated transformation and plant regeneration of the spring rape seed cultivar 'Precocity', using meristematic portions of hypocotyls as explants. The goal of this study was to establish an $A$. tumefaciens-mediated transformation procedure for B. napus hypocotyl segments to pave the way for the generation of transgenic plants.

\section{MATERIAL AND METHODS}

\section{Plant materials}

Rapeseeds (B. napus 'Precocity') were obtained from the Academy of Agricultural Sciences in Qinghai Province. The seeds were sterilized by incubation for $3 \mathrm{~min}$ in $70 \%$ alcohol, followed by $30 \mathrm{~min}$ in $15 \% \mathrm{H}_{2} \mathrm{O}_{2}$. The seeds were then soaked in sterilized water for $4 \mathrm{~h}$ and allowed to germinate on MS (Murashige and Skoog) medium containing 3\% sucrose $(\mathrm{w} / \mathrm{v})$ and $0.55 \%$ agar $(\mathrm{w} / \mathrm{v})$. Unless otherwise mentioned, the $\mathrm{pH}$ of the medium used in this study was approximately 5.8 . Plants were maintained at $25^{\circ}+1{ }^{\circ} \mathrm{C}$ under a $16 / 8$ cycle (light/ dark) using cool white fluorescent light.

\section{Culture conditions and plant regeneration}

Sterile seedlings were used as the source of explants for regeneration studies. Hypocotyl segments $(0.6$ to $1 \mathrm{~cm})$ were obtained from 5 to 10-day-old seedlings and tested for growth for a period on shoot induction media and shoot regeneration media containing the following hormonal combinations: a) Shoot induction media, 2,4-D (2,4-dichlorophenoxyacetic acid) (0.5 to $2 \mathrm{mg} / \mathrm{L}), 6$-BA (6-benzyladenine) $(0.2$ to $1 \mathrm{mg} / \mathrm{L})$; b) Shoot regeneration media, NAA (a-naphthaleneacetic acid) (0.1 to $1 \mathrm{mg} / \mathrm{L}), 6-\mathrm{BA}(1.5$ to $4.5 \mathrm{mg} / \mathrm{L})$.

After the shoots reached a length greater than $3 \mathrm{~cm}$, they were excised from the explants and transferred to rooting media. When the lengths of the roots reached approximately 3 to $5 \mathrm{~cm}$, the plantlets were removed from the culture vessels, washed with water to remove any adhering medium, and then transferred to pots. The plants were covered with plastic film and placed in growth chambers for 4 to 6 days and were then moved to a greenhouse after 10 days.

\section{Agrobacterium culture and vector construction}

A. tumefaciens strain GV3101, harboring the pCAMBIA1301 or pBI121 plasmid, was used for plant transformation. Due to the presence of a $S a c$ I restriction enzyme site in both the desired HSP22 sequence and in the T-DNA region of plasmid pBI121, the site was removed from $H S P 22$ by site-directed mutagenesis without changing the identity of the amino acid (Figure 1A). The T-DNA construct is shown in Figure 1B and C, and the primers used in this experiment are included in Table 1.

A single colony of A. tumefaciens was cultured on solid Luria Broth medium containing $50 \mathrm{mg} / \mathrm{L}$ gentamycin, rifampicin, and kanamycin at $28^{\circ} \mathrm{C}$. The single clone was then agitated at $200 \mathrm{rpm}$ in a shaker for approximately $24 \mathrm{~h}$. Subsequently, the bacterial cells were collected by centrifugation and resuspended in MS media. The final concentration was adjusted to an $\mathrm{OD}_{600}$ value of approximately 0.5 . 


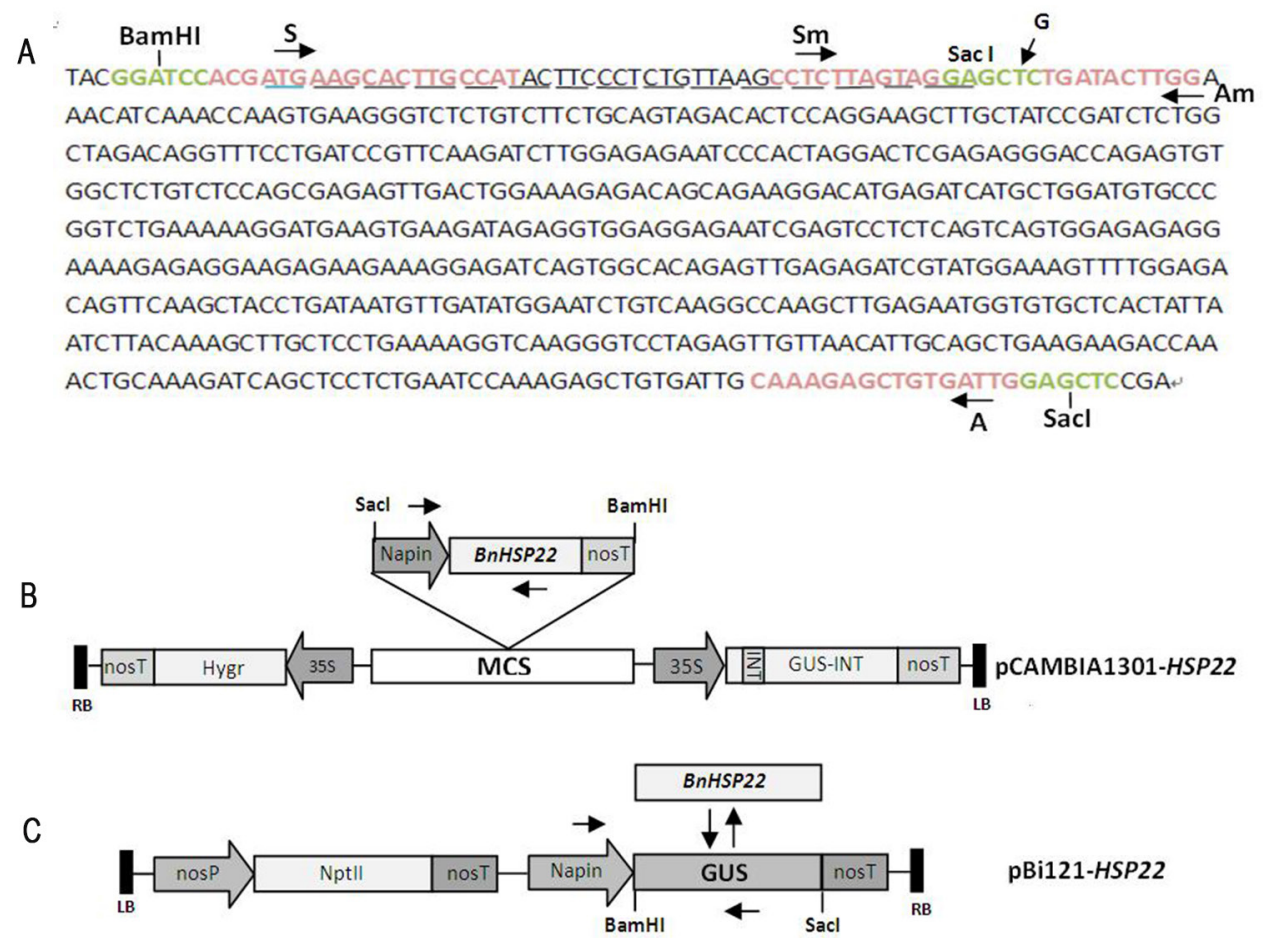

Figure 1. T-DNA constructs in vectors used for 'Precocity' cultivar transformation. A. Sequence of HSP 22 targeted by PCR site-directed mutagenesis to facilitate insertion into the pBI121 plasmid. Green and pink sequences indicate restriction enzyme sites and primer binding sites, respectively. B. Linear map of the T-DNA region of the recombinant pCAMBIA1301 plasmid. C. T-DNA region of pBI121-HSP22. Arrows indicate binding sites for different PCR primers used in this study.

\section{Table 1. Primers of the specific sequence amplified by PCR or qRT-PCR.}

\begin{tabular}{llll}
\hline \multirow{2}{*}{ Name } & \multicolumn{2}{c}{ Primer sequence 5' $\rightarrow 3^{\prime}$} & \multirow{2}{*}{ Function } \\
\cline { 2 - 3 } & Forward & Reverse & \\
\hline A & HSP22-F: CTCATCCCCCTTTTAAACCAACTT & HSP22-R: ATCTCTCAACTCTGTGCCACTGATC & \multirow{2}{*}{ Used for PCR } \\
& Vector-F: GCTAAAGGAGGCGGCATGGAA & Vector-R: CCCACGTCATAGAGCATCGGAAC & \\
B & S: TACGGATCCACGATGAAGCACTTGC & R: TCGGAGCTCCAATCACAGCTCTTTG & For overlap PCR \\
& Sm: CCTCTTAGTAGGAGCGCTGATACTTGG & Rm: CCAAGTATCAGCGCTCCTACTAAGAGG & \\
C & HSP22-F: GCTTGCTATCCGATCTGGCTAGA & HSP22-R: TCAACTCTGTGCCACTGATCTCCTT & \multirow{2}{*}{ Used for qPCR } \\
& $\beta$-Actin-F: TCTTCCTCACGCTATCCTCCG & $\beta$-Actin-R: AGCCGTCTCCAGCTCTTGC & \\
\hline
\end{tabular}

\section{Hygromycin sensitivity assay for hypocotyl segments}

The hygromycin sensitivity of hypocotyls subjected to transformation was tested in order to efficiently screen for transgenic plants after transformation. Hypocotyls excised from in vitro seedlings were cultured on shoot regeneration media containing $0.1 \mathrm{mg} / \mathrm{L}$ NAA, $2.5 \mathrm{mg} / \mathrm{L}$ 6-BA, 3\% sucrose, and $6.5 \mathrm{~g} / \mathrm{L}$ agar, supplemented with hygromycin $(0,2$, 
4, 6 , or $8 \mathrm{mg} / \mathrm{L}$ ). The explants were cultured at $25^{\circ} \mathrm{C}$ and sub-cultured at 2-week intervals. Hygromycin was added to the medium when it reached a temperature of approximately $55^{\circ} \mathrm{C}$ prior to solidification. After 20 days of culture, the optimal concentration of hygromycin was determined based on significant differences between the explants maintained under the conditions with different concentrations of hygromycin.

\section{Transformation of plant cells}

To produce transgenic plants, hypocotyls were immersed in bacterial suspension $\left(\mathrm{OD}_{600}\right.$ 0.5 ) for 3 to $5 \mathrm{~min}$. The explants were dried under a culture dish, on sterile filter paper, in order to remove excess bacteria, and were then cultured on co-cultivation medium containing shoot regeneration medium and acetosyringone. The plates were sealed with Parafilm and cultured under dark conditions at $25^{\circ} \mathrm{C}$ for 3 days. The hypocotyls were then transferred to selection medium, which was shoot regeneration media, supplemented with $4 \mathrm{mg} / \mathrm{L}$ hygromycin and $500 \mathrm{mg} / \mathrm{L}$ carbenicillin (Car). The explants cultured on shoot regeneration media served as the control. The selection medium was changed every 2 weeks in order to maintain selective pressure.

\section{Polymerase chain reaction (PCR) and quantitative real-time (qRT)-PCR analyses}

Total genomic DNA was extracted from young plant leaves using the cetyltrimethylammonium bromide (CTAB) method (Doyle, 1990). PCR $(25 \mu \mathrm{L})$ contained $12.5 \mu \mathrm{L}$ PCR mix, $2 \mu \mathrm{L}$ template DNA, and $0.5 \mu \mathrm{L}$ of each forward and reverse primers. DNA isolated from wild type plants served as a negative control, and the pCAMBIA-1301HSP 22 vector served as a positive control. The primer pairs were used to amplify fragments approximately $700 \mathrm{bp}$ in length that spanned a region from the napin promoter to HSP22. Reactions were incubated at $94^{\circ} \mathrm{C}$ for $5 \mathrm{~min}$, followed by 30 cycles of $30 \mathrm{~s}$ at $94^{\circ} \mathrm{C}, 30 \mathrm{~s}$ at a range of temperatures from $54^{\circ}$ to $58^{\circ} \mathrm{C}$, and $1 \mathrm{~min}$ at $72^{\circ} \mathrm{C}$, and a final extension at $72^{\circ} \mathrm{C}$ for $10 \mathrm{~min}$. Transgenic plants were identified, as previously described (Bubner and Baldwin, 2004), by qRT-PCR. First-strand cDNA was synthesized from $1 \mu \mathrm{g}$ RNA. The cDNA concentration was measured using a NanoDrop 1000 instrument (Thermo Fisher Scientific Inc., USA). Oligonucleotide primers were used to amplify a 230-bp fragment of HSP22. Rapeseed $\beta$-actin (AF111812) (Ling et al., 2006) was used as the reference gene for data normalization. A negative control lacking cDNA was included. qRT-PCR was conducted using the Mini Option Real-Time PCR system (Bio-Rad), following the manufacturer instructions. All reactions were conducted in sample wells containing $2 \mu \mathrm{L}$ cDNA, $10 \mu \mathrm{L}$ $2 \mathrm{X}$ SYBR Green I Master PCR mix, $1 \mu \mathrm{L} 0.5 \mu \mathrm{M}$ of each primer, and $6 \mu \mathrm{L}$ sterile water. Reactions were cycled under the following conditions: 5 min at $94^{\circ} \mathrm{C}, 25$ to 28 cycles of $94^{\circ} \mathrm{C}$ for $30 \mathrm{~s}$, annealing temperature $\left(59^{\circ} \mathrm{C}\right)$ for $30 \mathrm{~s}, 72^{\circ} \mathrm{C}$ for $1 \mathrm{~min}$, and a final extension at $72^{\circ} \mathrm{C}$ for $10 \mathrm{~min}$. Each reaction was performed in triplicate. All primer sequences are listed in Table 1. Primers were designed using the Primer Premier 6.0 software based on the identified rapeseed sequences.

\section{Histochemical $\beta$-glucoronidase (GUS) assay}

Transient GUS expression was analyzed in hypocotyl explants after co-cultivation 
on transformed and untransformed tissues. All explants were rinsed four times in doubledistilled water and were then stained in GUS solution overnight at $37^{\circ} \mathrm{C}$ as previously described (Jefferson, 1987). After staining, the explants were incubated in $70 \%$ ethanol until no chlorophyll remained. Transient GUS expression was calculated by recording the number of explants with blue staining.

\section{Statistical analysis}

Three replicates of 20 explants per treatment were analyzed to optimize the parameters of the A.tumefaciens-mediated transformation. Each experiment was repeated at least twice. The data were analyzed using Origin 8.0. One-way ANOVA was performed using the Statistical Package for the Social Sciences (SPSS) software.

\section{RESULTS AND DISCUSSION}

\section{Effect of plant growth regulators on regeneration of $B$. napus}

\section{Callus induction}

Callus induction is a critical step in plant regeneration. In this study, cotyledons and hypocotyls isolated from rapeseed were used to identify the optimal combinations of growth regulators. Explants were cultured on MS medium containing various combinations of cytokinin (6-BA) and auxin (2,4-D). A previous study showed that the combination of a higher concentration of auxin and a lower concentration of cytokinin was more efficient for induction of callus formation (Maheshwari et al., 2011). Similarly, results from the present study demonstrated that callus regeneration was minimal in the absence of 6-BA. When the minimum concentration $(0.2 \mathrm{mg} / \mathrm{L})$ of 6 -BA was added to the medium, the callus regeneration possessed the highest efficiency: $50.6 \%$ from hypocotyls and $29.3 \%$ from cotyledons. Upon increasing 6-BA concentration, callus regeneration in the explants gradually declined. This result was similar to that previously described by Maheshwari et al. (2011), which showed that increasing the concentration of BA in medium containing 2,4-D, decreased callus regeneration. We also analyzed combinations containing different concentrations of 2,4-D (Figure 2A and B). Addition of 2,4-D resulted in an increase in callus regeneration, and the optimal concentration was found to be $1 \mathrm{mg} / \mathrm{L}$, consistent with a previous study (Maheshwari et al., 2011). However, the effects of 2,4-D decreased at higher concentrations. In general, the combination of $1 \mathrm{mg} / \mathrm{L}$ auxin (2,4-D) and $0.2 \mathrm{mg} / \mathrm{L}$ cytokinin (6-BA) was found to be optimal for callus induction in both cotyledon (40.3\%) and hypocotyl (61.7\%) explants (Figure 2A and B).

\section{Shoot regeneration}

Due to the effect of age on physiological status, the age of the explant material is critical for shoot regeneration. In order to determine the optimal age for the explants, we tested explants cultured from 4-, 6-, 8-, 10-, and 13-day-old seedlings. Results from this analysis showed that 6 day-old seedlings were more beneficial for shoot regeneration (data not shown). The younger seedlings rarely exhibited callus responses, while the older seedlings generated more unorganized calli. 
A

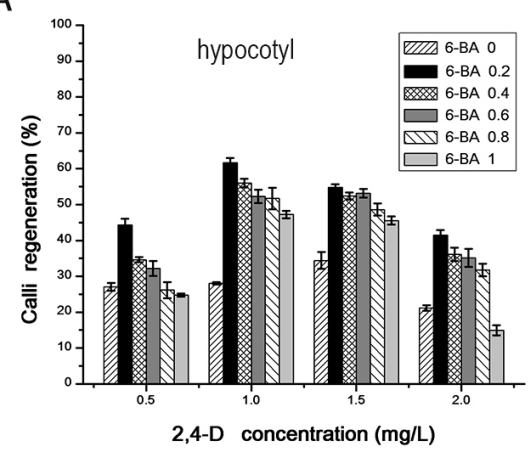

C

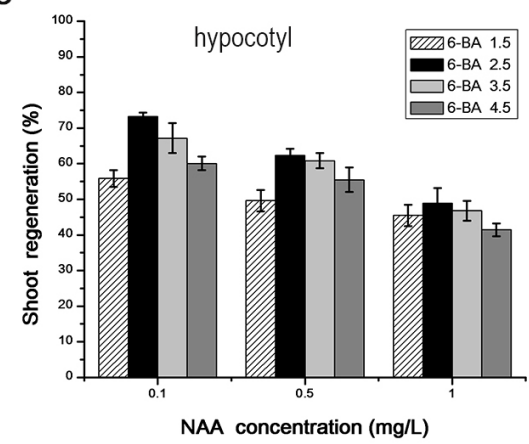

B

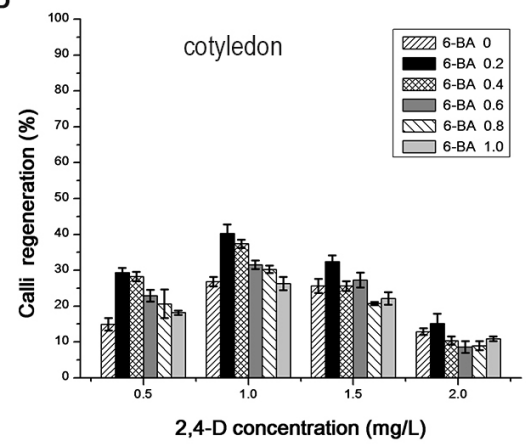

D

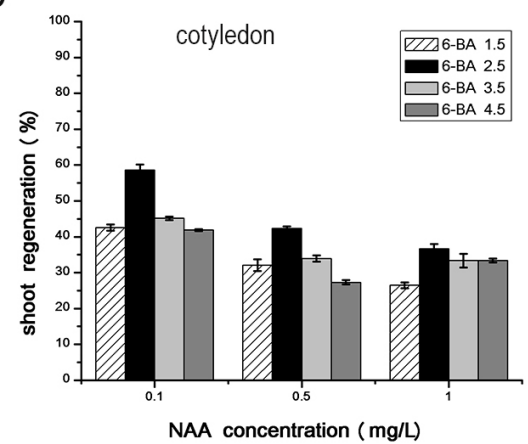

Figure 2. Callus or shoot regeneration from cotyledon and hypocotyl segments of plants on callus induction medium and shoot regeneration medium. Images on the left (A and $\mathbf{C})$ and right (B and $\mathbf{D})$ show regeneration of hypocotyl segments and cotyledon segments respectively. Thirty explants were cultured for each treatment, and the experiments were repeated at least twice. SD was calculated from 60 explants.

After callus induction, we attempted to identify the optimal concentrations of NAA and 6-BA for induction of shoot buds. An earlier study reported that high levels of auxin and cytokinin are essential for shoot regeneration from different types of explants. Ali et al. (2007) showed that media containing $0.1 \mathrm{mg} / \mathrm{L}$ NAA and $4.5 \mathrm{mg} / \mathrm{L} 6-\mathrm{BA}$ is beneficial for shoot regeneration of B. napus. In the present study, we found that medium supplemented with $0.1 \mathrm{mg} / \mathrm{L}$ NAA and $2.5 \mathrm{mg} / \mathrm{L}$ 6-BA was optimal for shoot regeneration from cotyledon and hypocotyl explants. The highest observed frequencies of shoot regeneration from cotyledon and hypocotyl segments were 57.9 and $73.3 \%$, respectively (Figure 2C and D). Other concentrations of NAA and 6-BA led to the production of more callus, as opposed to shoot regeneration.

Shoot induction and proliferation from cotyledons requires a longer time than that from hypocotyl segments, which regenerated in 4 weeks. The shoot regeneration frequency was approximately $18 \%$ higher for hypocotyls compared with cotyledons. Thus, we determined that hypocotyl was a better choice than cotyledon for further experiments regarding in vitro regeneration. Similarly, previous reports indicated that hypocotyls are better than cotyledons as explants for transformation experiments (Cardoza and Stewart, 2003; Maheshwari et al., 2011), although this comparative response is also related to genotype. 
In the present study, we found that the formation of shoots from cotyledon and hypocotyl differed in the shoot regeneration system compared with the transformation system. The shoot always regenerated directly from the explants in the plant tissue regeneration experiments (Figure 3). However, after infection by A. tumefaciens, a majority of both hypocotyl and cotyledon explants first produced callus, and then shoots regenerated from the calli; only a few shoots directly regenerated from the explants. Thus, incubation of explants with Agrobacterium appears to lead to callus induction cell by cell, and shoot buds subsequently regenerate from callus.
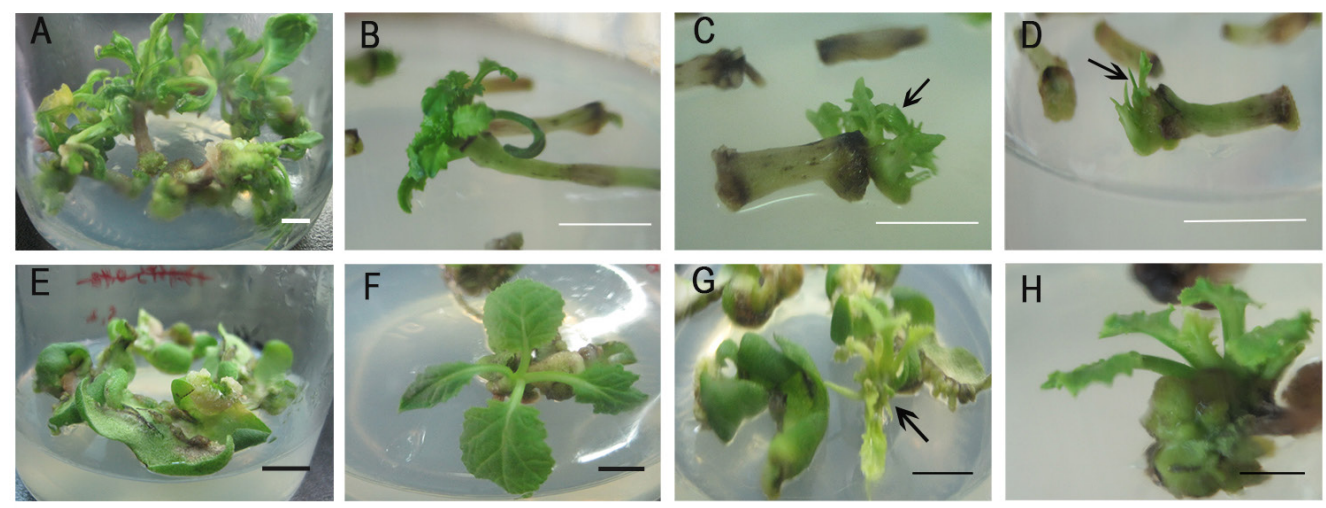

Figure 3. Shoot regeneration from the calli of cotyledons and hypocotyls or directly from Brassica napus explants ('Precocity' cultivar). A. B. Explants were cultured on shoot regeneration medium. C. Shoot regeneration direct from hypocotyl explants on selective media. D. E. Shoot regeneration from callus on selective medium. F. G. Shoot regeneration direct from explants on selective medium. H. Shoot regeneration from calli of cotyledon explants. Scale bars $=1 \mathrm{~cm}$.

\section{Effect of hygromycin on survival of hypocotyl segments}

In order to reduce loss of transgenic individuals, selection on hygromycin is necessary after transformation. Due to significant differences in hygromycin sensitivity between rape seed cultivars, we first analyzed hygromycin sensitivity of hypocotyl explants. Explants from seedlings of three different ages $(4,8$, and 12 day-old) were cultured on shoot regeneration media containing different concentrations of hygromycin (0, 2, 3, 4, 5, and $10 \mathrm{mg} / \mathrm{L})$. Explants cultured on hygromycin-free media served as the control, representing $100 \%$ survival. The frequency of the surviving explants drastically declined with increasing hygromycin concentrations ranging from 2 to $5 \mathrm{mg} / \mathrm{L}$ (Figure 4 ). Approximately $8.7 \%$ survival was obtained when the hygromycin concentration was increased to $4 \mathrm{mg} / \mathrm{L}$. These results are not consistent with those of Bhuiyan et al. (2011), who reported that $15 \mathrm{mg} / \mathrm{L}$ hygromycin was optimal for the primary selection of transgenic shoots. However, differences exist among various cultivars of B. napus. Therefore, based on our results, the selection procedure used for transformants in subsequent experiments was $4 \mathrm{mg} / \mathrm{L}$ hygromycin in order to maximize efficiency.

\section{Optimization of factors affecting GUS expression and hygromycin resistance}

Use of optimal biological conditions for the explants is important for maximizing 
infection and T-DNA transfer through A. tumefaciens. Many studies have reported a number of factors capable of influencing Agrobacterium-mediated transformation, including biotic and abiotic conditions such as regeneration, cultivar identity (Boszoradova et al., 2011), preconditioning time, co-cultivation time (Cardoza and Stewart, 2003; Zhao et al., 2013), and pre-selection period (Khan et al., 2003). Monitoring the function of visible reporter genes is crucial for selection of transgenic shoots, as well as for standardizing the factors affecting efficiency of transformation (Chattopadhyay et al., 2011). Using the pCAMBIA1301 binary vector, the CaMV35S (Cauliflower mosaic virus 35S) promoter was used to drive gusA expression. A modified intron inserted between the first and second exon of the gusA gene ensured that GUS activity occurred only after the T-DNA was transformed into plant cells, avoiding the influence of GUS expression in Agrobacterium (Chattopadhyay et al., 2011). Our results indicate that four key factors, seedling age, Agrobacterium concentration, infection time, and co-cultivation time, affect Agrobacterium-mediated transformation as revealed by GUS expression and hygromycin resistance.

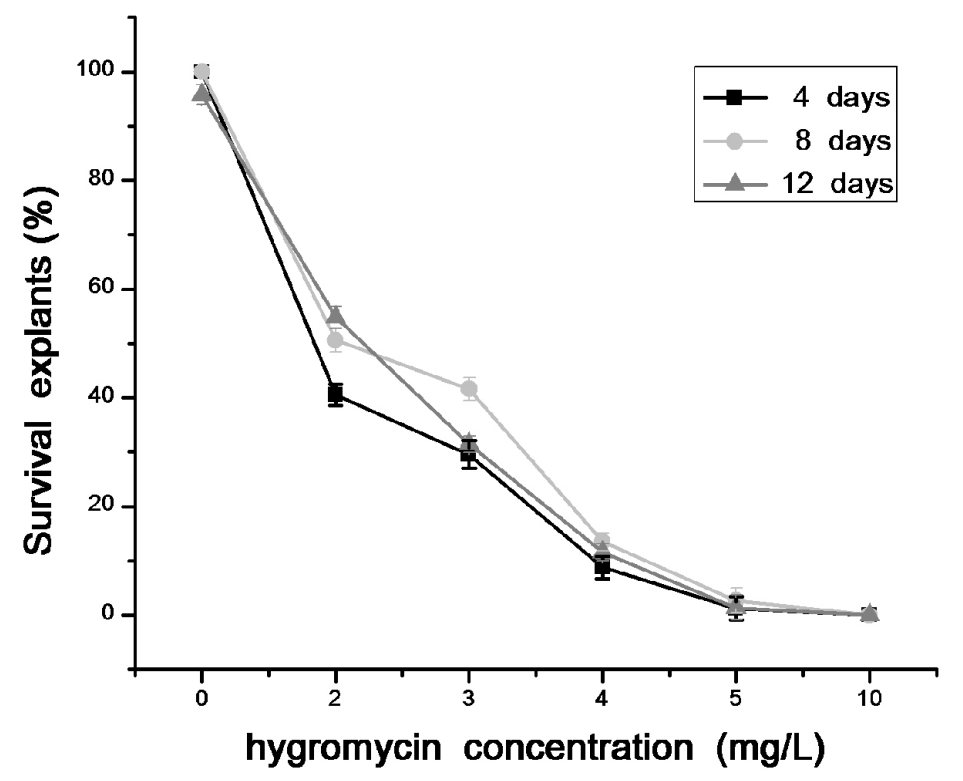

Figure 4. Effect of different concentrations of hygromycin on the survival of hypocotyls from Brassica napus ('Precocity' cultivar). The three lines represent seedlings of different ages (4, 8, and 12 days). Explants were cultured on shoot regeneration medium containing hygromycin $(0,2,3,4,5$, and $10 \mathrm{mg} / \mathrm{L})$. Data are reported as mean $\pm \mathrm{SE}$ of three independent experiments with 30 explants.

Previous studies have reported the use of seedlings of different ages from various plant species as donor material for transgenic experiments, including 8-day-old canola seedlings (Maheshwari et al., 2011) and 21-day-old B. napus seedlings (Jonoubi et al., 2005). In the present study, GUS expression and hygromycin resistance were monitored to evaluate the effect of explant age on the transformation frequency of the B. napus 'Precocity'. Hypocotyl segments derived from 4-, 6-, 8-, and 10-day-old seedlings were incubated for 3 to $5 \mathrm{~min}$ in bacterial suspension $\left(\mathrm{OD}_{600}=0.5\right)$. Results from this analysis revealed that explants from 
6- and 8-day-old seedlings exhibited the highest transformation frequency, as assessed by GUS expression (about 24\%) and hygromycin resistance (approximately 32\%) (Figure 5A). In survival response experiments, 6-, 8-, and 10-day-old seedlings cultured on selected media exhibited no significant differences, whereas 8-day-old seedlings exhibited more GUS expression than 6- and 10-day-old seedlings (Figure 5A). Differences in transformation frequency have previously been reported in B. napus plants of different seedling ages. Cardoza and Stewart (2003) found that seedlings less than 10 days old were optimal for shoot regeneration and transformation. Maheshwari et al. (2011) found that hypocotyls from 8-day-old seedlings were optimal for Agrobacterium-mediated transformation of B. napus. In addition, it was observed that 5-day-old seedlings of the rutabaga cultivar 'American Purple Top Yellow' increase transformation efficiency (Song et al., 2012). All these observations mentioned may primarily be related to a variety of endogenous plant hormones.

A

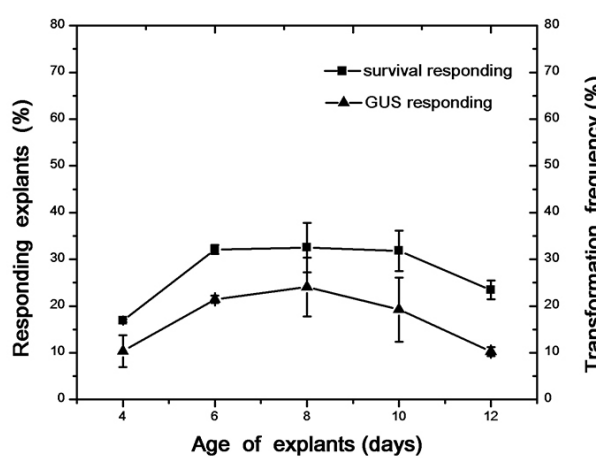

C

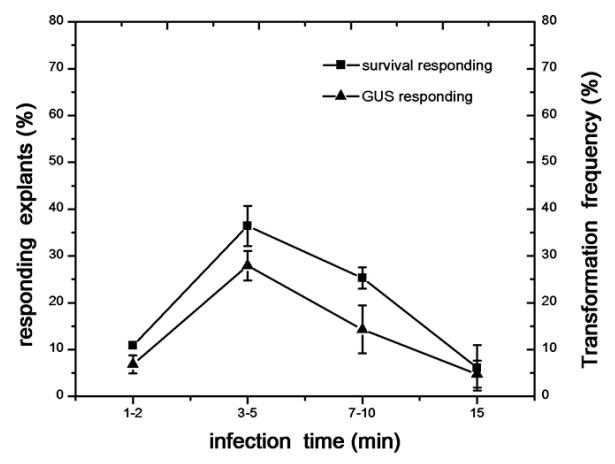

B

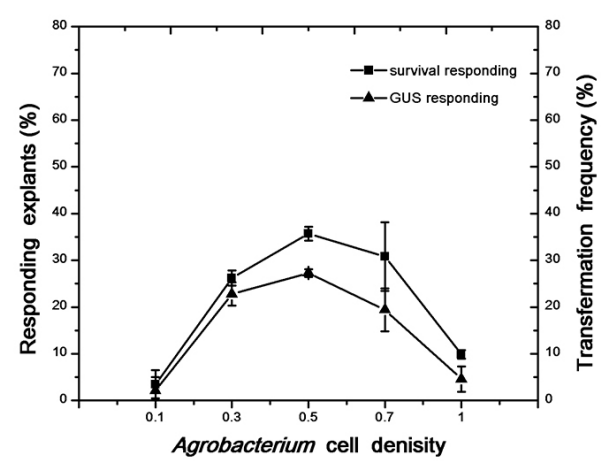

D

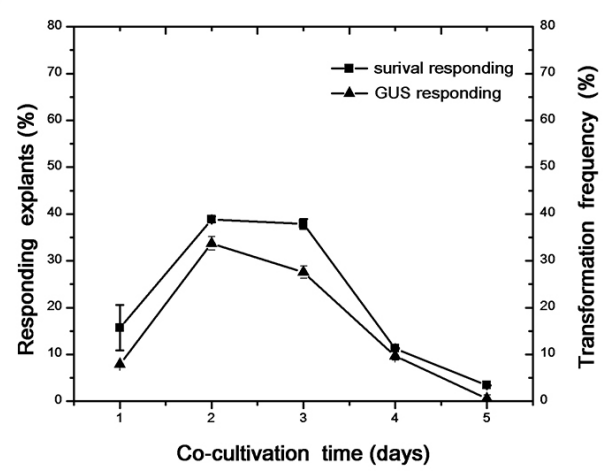

Figure 5. Effect of different factors on efficiency of transformation of explants. Squares represent the percentage of responding explants based on hygromycin resistance, and triangles represent the percentage of responding explants based on histochemical GUS expression. The transformation frequency was calculated as the number of resistant explants exhibiting GUS expression of total number of explants. All data are from plants grown on selective medium after 2 weeks of infection. Data are reported as mean \pm SE of three replicates of two experiments of 25 explants.

The influence of Agrobacterium concentration on plant transformation has been studied in many species, including jute (Corchorus capsularis L.) (Chattopadhyay et al., 2011), belladonna (Song and Walworth, 2013), sweet orange (Khan et al., 2012), and sunflower (Sujatha et al., 
2012). To identify the optimal concentration of $A$. tumefaciens required for high transformation frequency in rape seed, hypocotyl segments from 8-day-old seedlings were incubated in $50 \mathrm{~mL}$ media containing varying concentrations of Agrobacterium $\left(\mathrm{OD}_{600}=0.1,0.3,0.5,0.7\right.$, and 1). We observed a significant influence of bacterial concentration on transformation and regeneration from hypocotyls. The increase in Agrobacterium concentration up to $\mathrm{OD}_{600} 0.5$, resulted in concomitant increases in transformation frequency up to $27.27 \%$ by GUS expression and $35.71 \%$ by hygromycin resistance (Figure 5B). Few Agrobacterium cells were observed to enter plant cells in mixtures containing low concentrations of A. tumefaciens. However, with $\mathrm{OD}_{600}$ values above 0.5 , the transformation frequency gradually decreased, and most explants were observed to be necrotic. These results differ from the results of Cardoza and Stewart (2003), who reported that the optimum Agrobacterium concentration for transformation was $\mathrm{OD}_{600} 0.8$.

The duration of exposure of explants to Agrobacterium suspension is also a critical variable in the process of Agrobacterium-mediated transformation. Infection of Agrobacterium, transference, and T-DNA integration must all be completed in this period. We found that when incubated in Agrobacterium suspension, the explants immediately became inflexible. Explants from the optimal conditions were immersed in Agrobacterium cell suspensions for different time intervals (1, 3 to 5,7 to 10 , and $15 \mathrm{~min}$ ). The maximum transformation frequency based on shoot resistance $(36.4 \%)$ and GUS expression $(27.9 \%)$ occurred when the hypocotyl segments were incubated in Agrobacterium suspension for 3 to $5 \mathrm{~min}$, and this time was used in subsequent experiments. The transformation frequency declined with longer or shorter infection times (Figure 5C). The lower transformation frequency may be due to an insufficient number of Agrobacterium cells capable of interacting with hypocotyl segments over this period. In contrast, higher Agrobacterium concentrations with an overgrowth of Agrobacterium cells could lead to necrosis of the explants.

Co-cultivation time is another crucial factor affecting Agrobacterium-mediated gene transfer in plant cells. In this study, five time points $(1,2,3,4$, and 5 days) were investigated to determine the influence of co-culture period on transformation frequency, as analyzed by hygromycin resistance and GUS expression. Both hygromycin resistance and GUS expression were found to increase from 1 to 2 days of co-cultivation. The transformation frequency associated with 3 days of co-cultivation was slightly lower than that for 2 days, and drastic decreases were observed for times longer than 3 days (Figure 5D). These results are in agreement with a previous report stating that co-cultivation for 2 days yielded the highest transformation frequency (Cardoza and Stewart, 2003). In other studies, co-cultivation for three or four days has been used for transformation of $B$. napus. Thus, transformation efficiency likely depends on cultivar identity, explant type, physiological function, and other factors.

\section{Production of transgenic plants}

Chetty et al. (2013) reported the comparative efficacy of various Agrobacterium strains for plant transformation. However, few studies have shown that different vectors affect the transformation efficiency in plants, especially B. napus. Therefore, in this study, we assessed whether plasmid identity is a critical factor for Agrobacterium-mediated transformation of $B$. napus by comparing pCAMBIA1301 with pBI121. The results showed that $26.9 \%$ of explants transformed with Agrobacterium carrying pCAMBIA1301 $\left(\mathrm{OD}_{600} \sim 0.5\right)$ were resistant to hygromycin. In contrast, only $10.94 \%$ of explants were resistant to kanamycin after transformation with the same concentration of bacteria carrying the pBI121 plasmid 
(Table 2). It is likely that a single cell carries more pCAMBIA1301 plasmids than pBI121 plasmids, which would lead to increased T-DNA integration into the plantlet genome at the same Agrobacterium concentration. Thus, for subsequent experiments, we used Agrobacterium containing the pCAMBIA1301 plasmid for transformation experiments.

Table 2. Effect of different binary vectors used for genetic transformation of Brassica napus 'Precocity' cultivar hypocotyls.

\begin{tabular}{lccccc}
\hline Vector type & $\begin{array}{c}\text { Number of } \\
\text { explants evaluated }\end{array}$ & $\begin{array}{c}\text { Number of survival } \\
\text { callus/shoot }\end{array}$ & $\begin{array}{c}\text { Frequency of } \\
\text { response (\%) }\end{array}$ & $\begin{array}{c}\text { Number of shoot } \\
\text { regenerated }\end{array}$ & $\begin{array}{c}\text { Regeneration } \\
\text { frequency }(\%)\end{array}$ \\
\hline pBi121-HSP22 & 256 & 28 & 10.94 & 13 & 5.1 \\
pCAMBIA1301-HSP22 & 301 & 81 & 26.9 & 29 & 9.6 \\
\hline
\end{tabular}

${ }^{a}$ Frequency of response was defined as the number of survival explants selected on hygromycin / the number of total explants x $100 .{ }^{b}$ Regeneration frequency was calculated as the number of explants that regenerated shoot/total number of explants evaluated x 100. About 100 explants were cultured for both treatment and tested after 4 weeks of culture, and the data were calculated from three independent experiments.

After selecting the optimal parameters, hypocotyl segments from 8-day-old seedlings were incubated with Agrobacterium ( $\left.\mathrm{OD}_{600} 0.5\right)$ for 3 to $5 \mathrm{~min}$ and then co-cultured for 2 days. Three weeks later, GUS expression was examined in the explants using a histochemical assay. GUS expression was also analyzed in some of the regenerating shoots, which were cultured on selective media and clearly showed blue color, whereas non-transformed tissues exhibited no color change. Fully-grown regenerating plants were transferred to MS media lacking a carbon source to keep the plants strong, and were then cultured on rooting medium. Most plantlets initiated growth of 3 to 5 roots within three weeks. The well-rooted plantlets were transplanted to small pots, covered with plastic film to acclimate to the external environment, and then transferred to large plastic pots for subsequent growth (Figure 6).
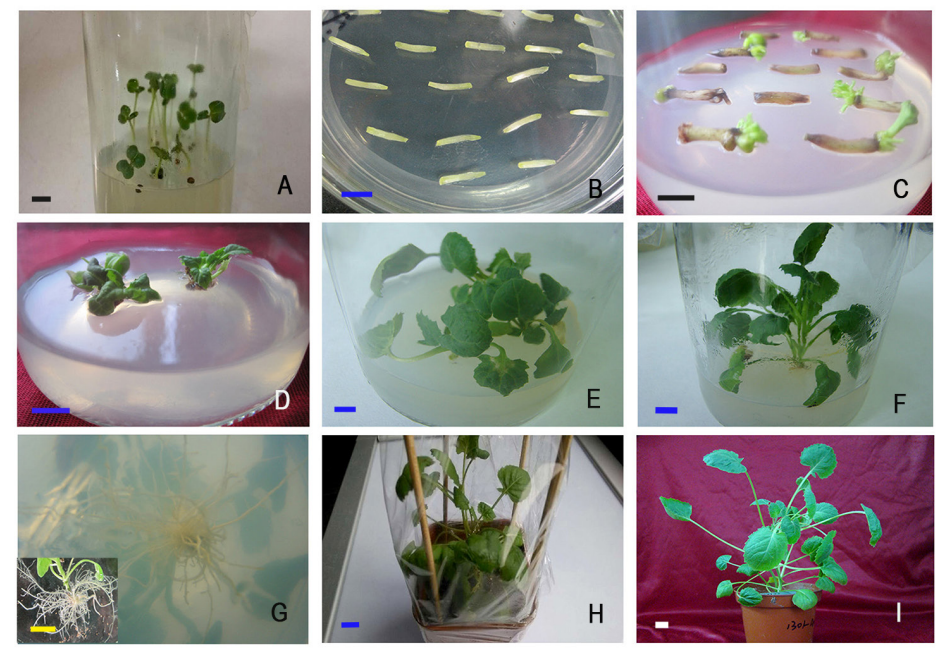

Figure 6. A. Seedlings cultured on MS basic medium. B. Precultured hypocotyls infected with Agrobacterium were cultured on co-cultivation medium. C. Callus or shoots of explants selected on medium containing hygromycin. D. E. Survival shoots subcultured on shoot selected medium. F. Regenerated plants domesticated on MS basic medium without sucrose. G. Plantlets transferred on rooting medium. H. I. Rooted plants transferred to soil. Scale bars $=1 \mathrm{~cm}$. 


\section{Root regeneration}

Ahmed et al. (1998) reported that root establishment in transgenic shoots requires a great deal of labor and time. A previous study reported that because half-strength media has reduced ionic strength, it is more beneficial for regenerated shoot rooting (George, 1996; Upreti and Dhar, 1996; Kooi et al., 1999). To identify the optimal medium for root formation, we tested thirteen medium compositions (Table 3). No differences in root regeneration were observed between plants grown in MS or $1 / 2$ MS in the present study. Both conditions led to no root production in 8 weeks. Rooting frequencies of approximately $20 \%$ were observed after the shoots were transplanted to media containing $0.1 \mathrm{mg} / \mathrm{L} \mathrm{NAA}$ and $200 \mathrm{mg} / \mathrm{L}$ Car. In contrast, rooting medium supplemented with Cefalexin (Cef) instead of Car resulted in increased shoot rooting. Rooting frequencies of $97.7 \%$ were obtained after the regenerated shoots were transferred to medium containing $0.5 \mathrm{mg} / \mathrm{L}$ indole butyric acid (IBA) and 200 $\mathrm{mg} / \mathrm{L}$ Cef. Adding a lower concentration of sucrose to the rooting media was more beneficial for root induction, consistent with previous studies (Kooi et al., 1999).

Table 3. Effect of different media on the root regeneration of in vitro regenerated shoots from seedling hypocotyls segments.

\begin{tabular}{lcc}
\hline Medium & Frequency of root regeneration (\%) & Time required for rooting initiation \\
\hline $1 / 2 \mathrm{MS}$ & 0 & - \\
MS & 0 & - \\
MS + 0.1 NAA + Car & 16.7 & $4-6$ weeks \\
MS + 0.5 NAA + Car & 6.7 & $4-6$ weeks \\
MS + 1 NAA + Car & 6.7 & $4-6$ weeks \\
MS + 0.1 NAA + Cef & 0 & - \\
MS + 0.5 NAA + Cef & 28.6 & 22 days \\
MS + 1 NAA + Cef & 14.3 & 30 days \\
MS + 0.1 IBA + Cef & 20.0 & $17-20$ days \\
MS + 0.5 IBA + Cef & 97.7 & $10-13$ days \\
MS + 1 IBA + Cef & 40.0 & $17-20$ days \\
\hline
\end{tabular}

Frequency of root regeneration was defined as the number of rooted plantlets out of total number of regenerated plants.

\section{Analysis of transgenic plants}

To confirm the integration of T-DNA, we used two pairs of primers, one pair specific for HSP 22 and one pair that binds outside the T-DNA region. In this manner, transgenic plants integrated with HSP 22 were selected, with a non-transformed plant and water used as controls (Figure 7). HSP22 was detected in the transgenic plants. However, these sequences were not amplified in non-transformed plants. Fragments outside the T-DNA were also not detected. These results indicate that transgenic plants over-expressing HSP22 were successfully acquired through Agrobacterium-mediated transformation.

Identifying the transgenic plants is a critical step. Southern blot analysis requires a large quantity of genomic DNA and requires a long time. Some studies have reported that qRTPCR analysis can be used as an alternative method for determining the transgenics as opposed to Southern blot analysis (Dutt et al., 2008, Zhao et al., 2013). Thus, in our study, we tested the transgenic $B$. napus 'Precocity' via qRT-PCR. These results showed that the transgenic plants analyzed have been transformed and resulted in the expression of HSP22 in this B. napus cultivar (Figure 8). 

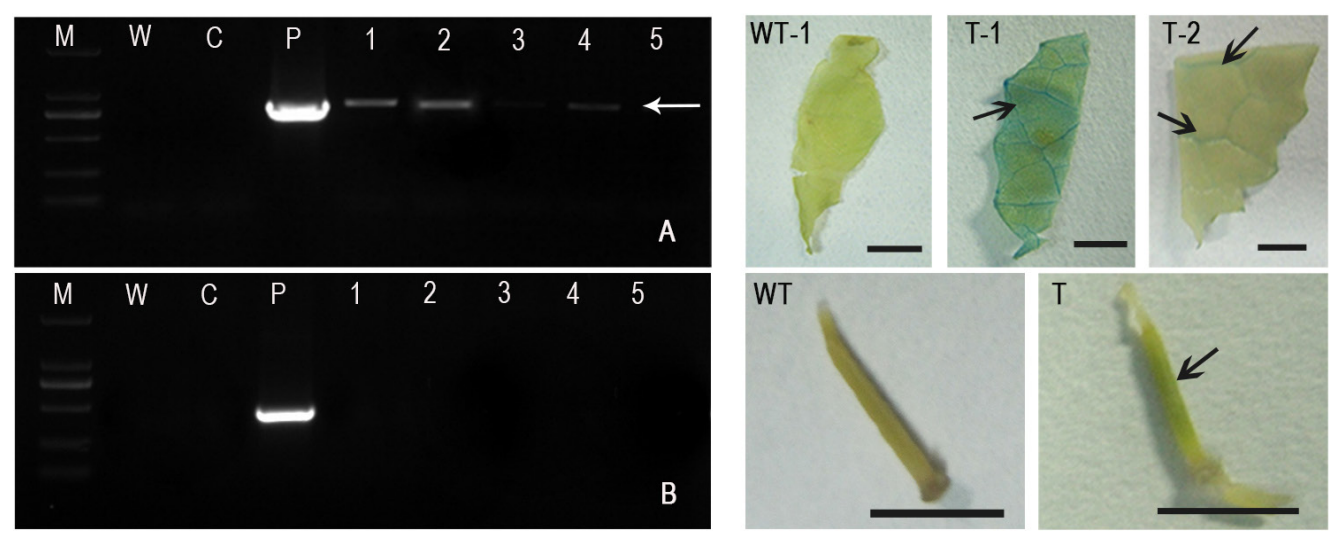

Figure 7. Analysis of transgenic plants by PCR and histochemical GUS expression. PCR was performed using primers specific for the T-DNA (between napin promoter and HSP22) (A) and a region outside of the T-DNA (B). lane $M=$ DL2000 DNA marker; lane $C=$ control untransformed plant; lane $P=$ positive control. Lanes $1-5$ contain products from transgenic plants. wt and wt- 1 represent wild type plants. t, t- 1 and $\mathrm{t}-2$ represent transgenic plants. The black arrows point to sites of GUS expression. Scale bars $=1 \mathrm{~cm}$.

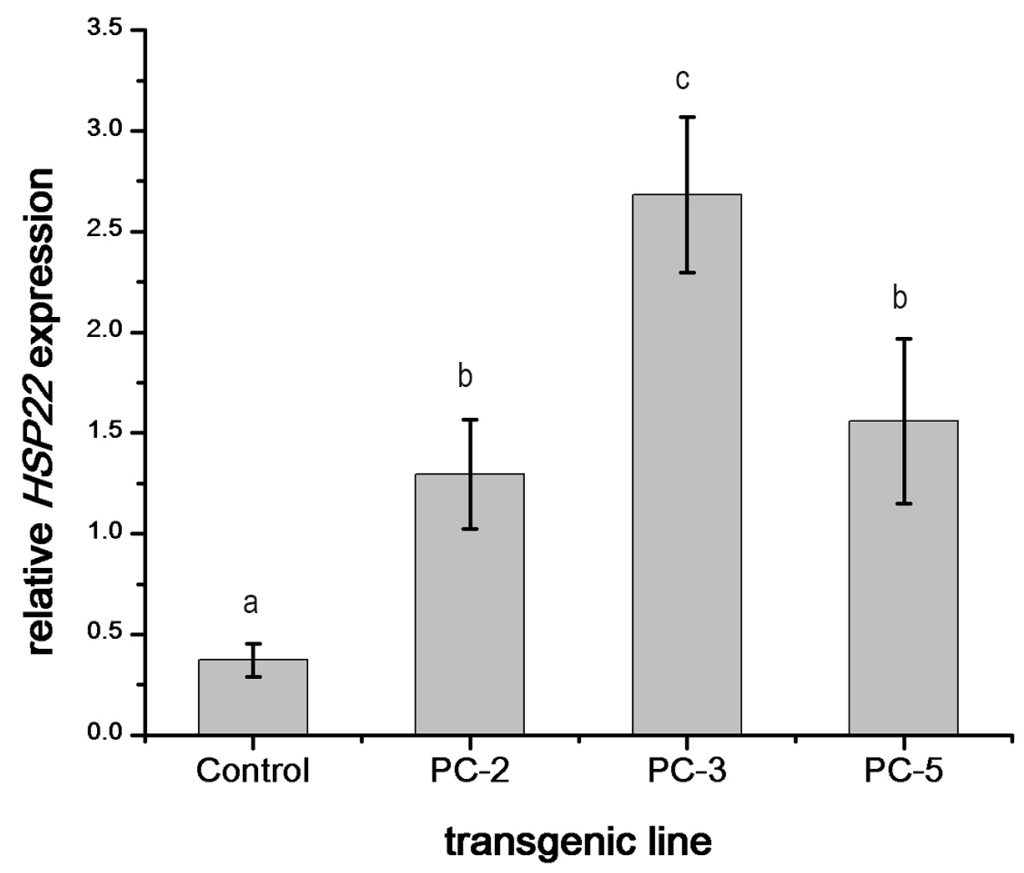

Figure 8. Qualitative and quantitative analyses of HSP22 expression in Brassica napus ('Precocity' cultivar). Relative expression levels of HSP22 transcripts in different lines (PC-2, PC-3, and PC-5) analyzed by qRT-PCR. Results were calculated as fold-changes relative to the control sample. Error bars represent standard error in gene expression. Numbers with different letters above the bars indicate significant differences by the Duncan multiple range test $(\mathrm{P} \leq 0.01)$. 
This study has established a successful system for shoot regeneration and Agrobacterium-mediated transformation from meristematic parts of hypocotyl explants of B. napus. The results show that media containing $1 \mathrm{mg} / \mathrm{L} \mathrm{2,4-D}$ and $0.2 \mathrm{mg} / \mathrm{L} 6-\mathrm{BA}$ was optimal for callus induction, which was followed by incubation in shoot regeneration medium supplemented with $0.1 \mathrm{mg} / \mathrm{L}$ NAA and $2.5 \mathrm{mg} / \mathrm{L}$ 6-BA. Transformation efficiency was shown to be influenced by many factors, including plant cultivar, type of explant tissue, strain and concentration of Agrobacterium, along with plasmid identity and other factors associated with the infection period. The operational protocols provide an alternative approach for regeneration of transgenic rapeseed and have potential for use in integrating other exogenous genes that may have significant value in the future.

\section{Conflicts of interest}

The authors declare no conflict of interest.

\section{ACKNOWLEDGMENTS}

Research supported by the Special Fund Project for the Scientific Research of the Forest Public Welfare Industry (\#201104024) and the National Natural Science Foundation of China (\#31271807), and the Academy of Agricultural Research Service in Qing Hai Province for seed resource.

\section{REFERENCES}

Abdollahi M, Corral-Martínez P, Mousavi A, Salmanian A, et al. (2009). An efficient method for transformation of pre-androgenic, isolated Brassica napus microspores involving microprojectile bombardment and Agrobacteriummediated transformation. Acta Physiol. Plantarum 31: 1313-1317.

Ahmed K, Khalique F and Malik B (1998). Modified artificial diet for mass rearing of chickpea pod borer, Helicoverpa (Heliothis) armigera (Hubn). Pak. J. Biol. Sci. 1: 183-187.

Ali H, Ali Z, Ali H, Mehmood S, et al. (2007). In vitro regeneration of Brassica napus L. cultivars (Star, Cyclone and Westar) from hypocotyls and cotyledonary leaves. Pak. J. Bot. 39: 1251-1256.

Bhalla PL and Smith N (1998). Agrobacterium tumefaciens-mediated transformation of cauliflower, Brassica oleracea var. botrytis. Mol. Breed. 4: 531-541.

Bhalla PL and Singh MB (2008). Agrobacterium-mediated transformation of Brassica napus and Brassica oleracea. Nat. Protoc. 3: 181-189.

Bhuiyan MSU, Min SR, Jeong WJ, Sultana S, et al. (2011). An improved method for Agrobacterium-mediated genetic transformation from cotyledon explants of Brassica juncea. Plant Biotechnol. 28: 17-23.

Boszoradova E, Libantova J, Matusikova I, Poloniova Z, et al. (2011). Agrobacterium-mediated genetic transformation of economically important oilseed rape cultivars. Plant Cell Tiss. Organ Cult. 107: 317-323.

Bubner B and Baldwin IT (2004). Use of real-time PCR for determining copy number and zygosity in transgenic plants. Plant Cell Rep. 23: 263-271.

Cardoza V and Stewart CN (2003). Increased Agrobacterium-mediated transformation and rooting efficiencies in canola (Brassica napus L.) from hypocotyl segment explants. Plant Cell Rep. 21: 599-604.

Chattopadhyay T, Roy S, Mitra A and Maiti MK (2011). Development of a transgenic hairy root system in jute (Corchorus capsularis L.) with gus A reporter gene through Agrobacterium rhizogenes mediated co-transformation. Plant Cell Rep. 30: 485-493.

Chetty VJ, Ceballos N, Garcia D, Narvaez-Vasquez J, et al. (2013). Evaluation of four Agrobacterium tumefaciens strains for the genetic transformation of tomato (Solanum lycopersicum L.) cultivar Micro-Tom. Plant Cell Rep. 32: 239-247.

Chhikara S, Chaudhary D, Yadav M, Sainger M, et al. (2012). A non-tissue culture approach for developing transgenic Brassica juncea L. plants with Agrobacterium tumefaciens. In Vitro Cell Dev. Biol. Plant 48: 7-14. 
Dhaka N, Chaudhary P and Sarin NB (2013). Agrobacterium mediated transformation using Glyoxylase II to increase salt tolerance in Gossypium hirsutum. Plant Mol. Biol. Biotechnol. 4: 1-5.

Doyle JJ (1990). Isolation of plant DNA from fresh tissue. Focus 12: 13-15.

Dutt M, Li ZT, Dhekney SA and Gray DJ (2008). A co-transformation system to produce transgenic grapevines free of marker genes. Plant Sci. 175: 423-430.

Fry J, Barnason A and Horsch RB (1987). Transformation of Brassica napus with Agrobacterium tumefaciens based vectors. Plant Cell Rep. 6: 321-325.

George E (1996). Plant propagation by tissue culture. Part 2: In practice. 2nd edn. Exegetics 1td., Basingstoke.

Halfhill MD, Richards HA, Mabon SA and Stewart CN (2001). Expression of GFP and Bt transgenes in Brassica napus and hybridization with Brassica rapa. Theor. Appl. Genet. 103: 659-667.

Hu Q, Andersen SB and Hansen LN (1999). Plant regeneration capacity of mesophyll protoplasts from Brassica napus and related species. Plant Cell Tissue Organ Cult. 59: 189-196.

Jefferson RA (1987). Assaying chimeric genes in plants: the GUS gene fusion system. Plant Mol. Biol. Rep. 5: 387-405.

Jonoubi P, Mousavi A, Majd A, Salmanian AH, et al. (2005). Efficient regeneration of Brassica napus L. hypocotyls and genetic transformation by Agrobacterium tumefaciens. Biol. Plantarum 49: 175-180.

Keshamma E, Sreevathsa R, Kumar AM, Reddy KN, et al. (2012). Agrobacterium-mediated in planta transformation of field bean (Lablab purpureus L.) and recovery of stable transgenic plants expressing the cry1 AcF gene. Plant Mol. Biol. Rep. 30: 67-78.

Khan EU, Fu XZ and Liu JH (2012). Agrobacterium-mediated genetic transformation and regeneration of transgenic plants using leaf segments as explants in Valencia sweet orange. Plant Cell Tissue Organ Cult. 109: 383-390.

Khan MR, Rashid H, Ansar M and Chaudry Z (2003). High frequency shoot regeneration and Agrobacterium-mediated DNA transfer in Canola (Brassica napus). Plant Cell Tissue Organ Cult. 75: 223-231.

Knutzon DS, Thompson GA, Radke SE, Johnson WB, et al. (1992). Modification of Brassica seed oil by antisense expression of a stearoyl-acyl carrier protein desaturase gene. Proc. Natl. Acad. Sci. U. S. A. 89: 2624-2628.

Konagaya K-I, Tsuda M, Okuzaki A, Ando S, et al. (2013). Application of the acetolactate synthase gene as a cisgenic selectable marker for Agrobacterium-mediated transformation in Chinese cabbage (Brassica rapa ssp. pekinensis). Plant Biotechnol. 30: 125-133.

Kong F, Mao S, Du K, Wu M, et al. (2011). Comparative proteomics analysis of OsNAS1 transgenic Brassica napus under salt stress. Chin. Sci. Bull. 56: 2343-2350.

Kooi LT, Keng CL and Hoe CTK (1999). In vitro rooting of sentang shoots (Azadirachta excelsa L.) and acclimatization of the plantlets. In Vitro Cell. Dev. Biol. Plant 35: 396-400.

Ling H, Zhao J, Zuo K, Qiu C, et al. (2006). Isolation and expression analysis of a GDSL-like lipase gene from Brassica napus L. J. Biochem. Mol. Biol. 39: 297-303.

Maheshwari P, Selvaraj G and Kovalchuk I (2011). Optimization of Brassica napus (canola) explant regeneration for genetic transformation. N. Biotechnol. 29: 144-155.

Mashayekhi M, Shakib AM, Ahmad-Raji M and Bezdi KG (2008). Gene transformation potential of commercial canola (Brassica napus L.) cultivars using cotyledon and hypocotyl explants. Afr. J. Biotechnol. 7: 4459-4463.

Moghaieb RE, El-Awady MA, El Mergawy RG, Youssef SS, et al. (2006). A reproducible protocol for regeneration and transformation in canola (Brassica napus L.). Afr. J. Biotechnol. 5: 143-148.

Pua EC, Mehrapalta A, Nagy F and Chua NH (1987). Transgenic plants of Brassica napus L. Biotechnology 5: 815-817.

Song GQ and Walworth A (2013). Agrobacterium tumefaciens-mediated transformation of Atropa belladonna. Plant Cell Tissue Organ Cult. 115: 107-113.

Song GQ, Walworth AE and Hancock JF (2012). Agrobacterium tumefaciens-mediated transformation of rutabaga (Brassica napus var. napobrassica) cultivar “American Purple Top Yellow”. In Vitro Cell Dev. Biol. Plant 48: 383-389.

Sujatha M, Vijay S, Vasavi S, Reddy PV, et al. (2012). Agrobacterium-mediated transformation of cotyledons of mature seeds of multiple genotypes of sunflower (Helianthus annuus L.). Plant Cell Tissue Organ Cult. 110: 275-287.

Upreti J and Dhar U (1996). Micropropagation of Bauhinia vahlii Wight \& Arnott - a leguminous liana. Plant Cell Rep. 16: $250-254$

Zhang Y and Bhalla PL (2004). In vitro shoot regeneration from commercial cultivars of Australian canola (Brassica napus L.). Aust. J. Agric. Res. 55: 753-756.

Zhang Y, Singh MB, Swoboda I and Bhalla PL (2005). Agrobacterium-mediated transformation and generation of male sterile lines of Australian canola. Aust. J. Agric. Res. 56: 353-361.

Zhao J, Li ZT, Cui J, Henny RJ, et al. (2013). Efficient somatic embryogenesis and Agrobacterium-mediated transformation of pothos (Epipremnum aureum) 'Jade'. Plant Cell Tissue Organ Cult. 114: 237-247. 